\title{
Satisfaction des utilisateurs du dossier patient informatisé valaisan
}

\author{
Dr Alex Gnaegia, \\ Dr Paul Cohena, \\ David Mareyb, \\ Matthieu Rivron ${ }^{b}$, \\ Dr Philippe Wieser ${ }^{\mathrm{b}}$ \\ anstitut Central \\ des Hôpitaux Valaisans, \\ Service d'informatique médicale \\ et administrative du Valais, Sion \\ ${ }^{b}$ Ecole Polytechnique Fédérale \\ de Lausanne, Institut international \\ de management pour la logistique, \\ Lausanne
}

\section{Summary}

A multidisciplinary and shared computerized patient record (CPR) has been progressively implemented since 2002 for all public hospitals in the State of Valais, Switzerland. This paper presents the results of a formal evaluation of user's satisfaction made during the deployment and based on questionnaires and interviews. According to the results, the major objectives have been reached: $2 / 3$ of users evaluate positively the effect of the CPR on access to patient information as well as improvement of communication between care providers. The performance problems are clearly identified, such as time needed to start the software. These problems govern the final adoption of the system, although $90 \%$ of users would not get back to paper. In conclusion, intrinsic quality of the CPR, such as ergonomics and structure, and technical aspects such as speed and availability are key factors for the success of such a deployment.

\section{Objectifs}

En 2000, sur la base d'un rapport de l'Ecole Polytechnique Fédérale de Lausanne [1], l'Etat du Valais décide de lancer un projet d'informatisation de l'ensemble des hôpitaux publics valaisans, nommé projet Infoval. Parmi les étapes du projet figure notamment la mise en place d'un dossier patient informatisé (DPI) multidisciplinaire et unique pour tous les hôpitaux. Basé sur un progiciel commercialisé, le DPI est un outil de travail commun pour les médecins, personnels soignants, physiothérapeutes et pharmaciens cliniques. En remplacement du dossier «papier», il permet la saisie de l'information et l'accès à celle-ci depuis n'importe quel ordinateur des hôpitaux sous réserve des droits d'accès. Il comporte un dossier médical (anamnèse, examen clinique, notes de suite, prescription de médicaments, ordres médicaux, lettres de sortie, résultats de laboratoire, etc.) et un dossier infirmier (anamnèse, processus de soins, feuille de surveillance des signes vitaux, distribution des médicaments). La structure du dossier patient est identique pour l'ensemble des établissements, mais respecte les spécificités des disciplines médicales. La mise en place du DPI s'est effectuée depuis l'automne 2002 progressivement service par service, mais chaque fois en incluant tous les corps de métiers (médecins, infirmières, physiothérapeutes, pharmaciens). En été 2005, le DPI est en production dans les services de médecine interne, chirurgie, réadaptation et gériatrie des hôpitaux de Brigue, Viège, Sierre, Sion, Martigny, ainsi qu'au Centre valaisan de pneumologie de Montana et à la clinique St-Amé à St-Maurice.

L'implémentation d'un dossier patient informatisé nécessite des investissements financiers et humains importants. Parvenu aux deux tiers du déploiement intégral, une étude sur l'impact du DPI sur l'organisation des hôpitaux et ses conséquences auprès des utilisateurs semble pertinente. Elle doit permettre d'objectiver la position des utilisateurs vis-à-vis du DPI et d'éviter de réagir uniquement sur la base des remarques des utilisateurs les plus négatifs ou les plus revendicatifs

\section{Méthodes}

L'évaluation s'est effectuée aux travers de deux méthodes: des entretiens ciblés d'utilisateurs ou d'intervenants du projet, ainsi qu'au moyen d'un questionnaire pour tous les utilisateurs via l'Internet.

Entretiens: les entretiens ont été menés par deux personnes étrangères au projet $(D$. Marey et $\mathrm{M}$. Rivron) et ont abordé les thèmes principaux suivants: sécurité et confidentialité des données, qualité du logiciel, impact sur l'activité du clinicien.

Questionnaire: il était composé de 44 questions principales, disponible en français et en allemand (pour les hôpitaux de Brigue et Viège) sur un site Internet. Les aspects suivants étaient examinés: profil de l'utilisateur, qualité du logiciel, pannes et support aux utilisateurs, impact du DPI sur le fonctionnement des services hospitaliers, sécurité et confidentialité des données. Toutes les questions étaient fermées, c'est-à-dire sans possibilité de saisie de texte libre de manière à faciliter l'interprétation des réponses. L'ensemble des utilisateurs du DPI ont été invités durant une semaine (mai 2005) par un message à l'ouverture 
du logiciel à se rendre sur un site Internet pour remplir le questionnaire de manière anonyme.

\section{Résultats}

Entretiens: 12 personnes ont été interrogées dont 7 médecins, 4 infirmiers et 1 pharmacien, provenant des hôpitaux de Martigny, Sion, Sierre,

Viège, du Centre valaisan de pneumologie et de l'Institut central des hôpitaux valaisans. Les principales conclusions sont les suivantes:

Sécurité et confidentialité des données: la confidentialité des données et des informations est aussi bien préservée, si ce n'est mieux qu'avec le dossier papier. Les ordres sont plus précis, mais la procédure est plus contraignante.

Qualité du logiciel: en dehors du module des graphiques des signes vitaux jugé peu pratique, le logiciel est estimé dans son ensemble complet et assez convivial. Il manque par contre certains liens entre les différents formulaires, ce qui engendre des saisies redondantes d'information. Par contre, des lenteurs dans l'accès aux données sont relevées par la quasi-totalité des personnes interrogées.

Impact sur l'activité du clinicien: la rigueur qu'apporte le logiciel dans la gestion des dossiers médicaux est la qualité principale reconnue par l'ensemble des acteurs. Le DPI apporte une vision globale sur tous les patients et génère moins de déplacements physiques des médecins grâce l'accès facilité à l'information. De plus, il harmonise le contenu des dossiers médicaux dans tout le canton et rend ceux-ci plus cohérents. Au sujet des éventuels gains de temps perçus par les utilisateurs, il se dégage une ambivalence, perte de temps à la saisie, mais gain de temps à la consultation.

Questionnaire: à partir des 2300 utilisateurs, 145 questionnaires ont été remplis, qui correspond à un taux de réponse de $15.8 \%$ avec un taux d'erreur d'échantillonnage de $8.14 \%$. La répartition entre les corps de métiers est présentée dans le tableau 1.

Qualité du logiciel: le temps d'ouverture du logiciel et/ou de réactivité à l'utilisation est jugé moyen par un tiers des utilisateurs, et défavorable par 30\% (ouverture) et respectivement $41 \%$ (réactivité) d'entre eux. En revanche l'ergonomie du logiciel remporte $42 \%$ d'avis positifs contre $24 \%$ d'avis négatifs.

Panne et support aux utilisateurs: la fréquence d'indisponibilité du DPI est évaluée à une fois
Tableau 1.

Profil des répondants au questionnaire.

\begin{tabular}{ll}
\hline & Répartition en $\%$ \\
\hline Médecin & 19,8 \\
\hline Infirmier/ère & 57,3 \\
\hline Technicien/ne en radiologie & 0,0 \\
\hline Physiothérapeute & 5,3 \\
\hline Diététicien/ne & 1,5 \\
\hline Ergothérapeute & 0,8 \\
\hline Pharmacien/ne & 3,8 \\
\hline Secrétaire médicale & 8,4 \\
\hline Personnel administratif & 3,1 \\
\hline
\end{tabular}

par semaine voire plus par la moitié des utilisateurs. Ce chiffre peut être considéré comme élevé mais il ne nous est par ailleurs pas possible de le confirmer ou l'infirmer. En effet, comme la disponibilité du DPI est conditionnée par tous les éléments de la chaîne informatique, à savoir ordinateur personnel, réseau, serveurs de présentation et serveurs de base de données, il suffit d'une défaillance d'un des éléments pour que le service soit jugé indisponible par l'utilisateur. Le support aux utilisateurs est effectué au sein du service informatique, il est jugé comme rapide par $9 \%$ des utilisateurs, suffisamment rapide par $34 \%$ et moyennement rapide par 38\%. Par contre la qualité du support est mieux évaluée puisqu' elle est jugée de très bonne qualité par $12 \%$ des répondants au questionnaire, et de bonne qualité par $62 \%$ d'entre eux.

Impact du DPI sur le fonctionnement des services hospitaliers: l'une des raisons principales de la mise en place d'un DPI est de faciliter l'échange d'information entre professionnels de la santé. En ce sens, l'objectif a été atteint car l'impact du DPI sur les échanges d'information avec les médecins a été jugé positif par près de deux tiers des utilisateurs (62\% pour les communications interhospitalières et $64 \%$ intrahospitalières), et de manière encore plus marquée avec les infirmières (68\% pour les communications interhospitalières et $74 \%$ intrahospitalières). Pour la majorité des utilisateurs qui ont émis un avis (toute catégorie confondue), le DPI apporte un gain de temps pour l'obtention des résultats de laboratoire, la recherche de dossiers, la recherche d'information sur les médicaments, l'accès aux rapports des consultants et les saisies administratives telles que lettre de sortie. 
Sécurité et confidentialité des données: il y a autant d'avis positifs que négatifs. En effet, 34\% des utilisateurs jugent favorablement l'impact du DPI sur la sécurité et la confidentialité des informations médicales, alors qu'également 34\% d'entre eux ont un avis défavorable. Par contre, le DPI a un effet positif sur la qualité et la quantité des données saisies (46\% d'avis favorables contre $20 \%$ d'avis défavorables).

Enfin, près de $60 \%$ de la population sondée estime que l'informatisation doit être étendue et appliquée à la médecine ambulatoire ainsi qu'aux établissements médico-sociaux (EMS) et centres médicosociaux (CMS). Seul 15\% des opinions ne vont pas dans ce sens. En réponse à la demande sur les améliorations qui feraient définitivement adopter le dossier électronique, les problèmes de performances du logiciel (délai à l'ouverture et à l'utilisation) ressortent clairement comme la doléance principale, quand bien même $86 \%$ des sondés n'envisagent pas de retour au dossier papier.

Nous relevons l'intérêt de procéder à une évaluation de la satisfaction des utilisateurs au moyen de deux méthodes. En effet, les entretiens per- mettent d'obtenir une vision précise et de bien comprendre les attentes et critiques des utilisateurs. Mais le risque est grand que les conclusions soient biaisées par le choix des personnes interviewées. Or, la deuxième méthode, soit l'enquête par questionnaire, permet justement de valider les principales tendances qui se dégagent des entretiens grâce au nombre significatif d'avis d'utilisateurs.

\section{Conclusion}

La mise en place d'un dossier patient informatisé pour l'ensemble des hôpitaux valaisans facilite l'accès à l'information en apportant une vision globale sur le dossier du patient et ce pour tous les professionnels de la santé. Des gains de temps sont relevés par les utilisateurs dans plusieurs activités quotidiennes. Si les qualités intrinsèques du logiciel (ergonomie, structure) et de sa paramétrisation sont importantes, la réussite du déploiement d'un dossier patient électronique est également conditionnée par les aspects techniques, en particulier la rapidité et la disponibilité de celui-ci.

\section{Références}

1 Wieser P. Informatisation des hôpitaux valaisans. Lausanne (Suisse). Ecole Polytechnique Fédérale de Lausanne; 2000. 